\title{
El Entorno laboral y la nutrición del personal de enfermería en áreas hospitalarias
}

\author{
The work environment and the nutrition of the nursing staff in hospital areas
}

\section{O ambiente de trabalho e nutrição da equipe de enfermagem em áreas hospitalares}

\author{
María Agustina Tapia Mieles ${ }^{1}$ \\ maria.tapia@uleam.edu.ec \\ https://orcid.org/0000-0003-1573-6689
}

\author{
Manuel Rich Ruiz ${ }^{2}$ \\ 1rirum@uco.es \\ https://orcid.org/0000-0003-3317-267X
}

\author{
${ }^{1}$ Universidad Laica Eloy Alfaro de Manabí, Manabí-Ecuador \\ ${ }^{2}$ Universidad de Córdoba- Córdoba-España
}

Recibido 22 de marzo 2021 | Arbitrado y aceptado 10 de abril 2021 | Publicado en 4 de mayo 2021

\begin{abstract}
RESUMEN
El presente estudio analiza los factores que constituyen el ambiente laboral hospitalario en relación con las dificultades en la nutrición del personal de enfermería. Objetivo. Analizar los aportes de investigaciones en el entorno laboral y sus repercusiones en el estado de salud nutricional del personal de enfermería en las áreas hospitalarias. Metodología. Metaanálisis a través de la aplicación de la declaración PRISMA (Preferred Reporting Items for Sistematycal Reviews and Meta-Analyses) para una revisión sistemática e integradora de las investigaciones seleccionadas. Se realizó una búsqueda en las principales bases de datos de Medicina y Ciencias de la Salud como: PubMed, Medline, SCOPUS, Wed of Science (Wos), Cinahl y Cochrane, con la cual se identificaron los artículos con pertinencia al ambiente laboral hospitalario, calidad nutricional, personal de enfermería. Se fundamentó la búsqueda con las preguntas Pico: ¿Cuál es la relación entre un entorno laboral y la nutrición del personal de enfermería del área hospitalaria? Cómo afecta el estrés y la fatiga laboral a la nutrición del personal de enfermería que se desempeña en el área hospitalaria P: Personal de enfermería del área hospitalaria; I: Entorno laboral; C: Estado nutricional. Se incluyeron en esta revisión 17 artículos. Se identificaron siete temas: sobrepeso y obesidad, actividad física, horarios de trabajo por turnos rotativos y nocturnos, hábitos y estilos de vida, conductas alimentarias, angustia mental y estrés laboral. Conclusión. Los factores negativos del ambiente laboral afectan la nutrición y conlleva al deterioro de la salud del personal de enfermería que trabaja en el área hospitalaria.
\end{abstract}

Palabras clave: Nutrición; sobrepeso; personal de enfermería; ambiente laboral hospitalario
MT: Enfermera profesional. Master en Gerencia en Salud para el desarrollo Local. Gerencia en Salud para el desarrollo Local. Reconocimiento por el SENESCY como profesor investigador Auxiliar 1 - REG-INV18-02342. Doctorando en Biociencias, Universidad de Córdoba España. Universidad Laica Eloy Alfaro de Manabí, Ecuador.

MR: Diplomado en Enfermería. Licenciado en Humanidades. Doctor por la Universidad de Córdoba. Profesor titular y director del Departamento de Enfermería, Farmacología y Fisioterapia de la Universidad de Córdoba. Integrante del CIBER de Fragilidad y Envejecimiento Saludable CIBERFES. Universidad de Córdoba, España.

\begin{abstract}
This study analyses the factors that constitute the hospital work environment in relation to difficulties in nutrition among nursing staff. Objective. To analyse the contributions of research on the work environment and its repercussions on the nutritional health status of nursing staff in hospital areas. Methodology. Meta-analysis through the application of the PRISMA statement (Preferred Reporting Items for Systematic Reviews and Meta-Analyses) for a systematic and integrative review of the selected research. A search was carried out in the main databases of Medicine and Health Sciences such as: PubMed, Medline, SCOPUS, Wed of Science (Wos), Cinahl and Cochrane, with which articles with relevance to the hospital work environment, nutritional quality and nursing staff were identified. The search was based on the following questions: What is the relationship between the work environment and the nutrition of hospital nurses? How does work stress and fatigue affect the nutrition of hospital nurses P: Hospital nurses; I: Work environment; C: Nutritional status. Seventeen articles were included in this review. Seven themes were identified: overweight and obesity, physical activity, rotating and night shift work schedules, habits and lifestyles, eating behaviours, mental distress and work stress. Conclusion. The negative factors of the work environment affect nutrition and lead to the deterioration of the health of nurses working in the hospital area.
\end{abstract}

Key words: Nutrition; overweight; nursing staff; hospital work environment 
MT: Enfermera profesional. Master en Gerencia en Salud para el desarrollo Local. Reconocimiento por el SENESCY como profesor investigador Auxiliar $1-\mathrm{REG}-\mathrm{NV}-$ 18-02342. Doctorando en Biociencis, 18-02342. Doctorando en Biociencias, Universidad de Córdoba España. Universidad Laica Eloy Alfaro de Manabí Ecuador.

MR: Diplomado en Enfermería. Licenciado en Humanidades. Doctor por la Universidad de Córdoba. Profesor titular y director del Departamento de Enfermería, Farmacología y Fisioterapia de la Universidad de Córdoba. Integrante del CIBER de Fragilidad Envejecimiento Saludable CIBERFES Universidad de Córdoba, España.

\begin{abstract}
RESUMO
0 presente estudo analisa os fatores que constituem o ambiente de trabalho hospitalar em relação às dificuldades na nutrição do pessoal de enfermagem. Objetivo. Analisar as contribuições da investigação no ambiente de trabalho e as suas repercussões no estado de saúde nutricional do pessoal de enfermagem nas áreas hospitalares. Metodologia. Meta-análise através da aplicação da declaração PRISMA (Preferred Reporting Items for Systematic Reviews and Meta-Analyses) para uma revisão sistemática e integradora da investigação selecionada. Foi efetuada uma pesquisa nas principais bases de dados de Medicina e Ciências da Saúde tais como: PubMed, Medline, SCOPUS, Wed of Science (Wos), Cinahl e Cochrane, com a qual foram identificados os artigos com relevância para o ambiente de trabalho hospitalar, qualidade nutricional, pessoal de enfermagem. A pesquisa foi baseada nas seguintes questões: Qual é a relação entre um ambiente de trabalho e a nutrição dos enfermeiros hospitalares? Como é que o stress e a fadiga no trabalho afetam a nutrição dos enfermeiros hospitalares P: Enfermeiros hospitalares; I: Ambiente de trabalho; C: Estado nutricional. Dezessete artigos foram incluídos nesta revisão. Foram identificados sete temas: excesso de peso e obesidade, atividade física, horários de trabalho rotativos e noturnos, hábitos e estilos de vida, comportamentos alimentares, angústia mental e stress laboral. Conclusão. Os fatores negativos do ambiente de trabalho afetam a nutrição e conduzem à deterioração da saúde dos enfermeiros que trabalham na área hospitalar.
\end{abstract}

Palavras-chave: Nutrição; excesso de peso; pessoal de enfermagem; ambiente de trabalho hospitalar

\section{INTRODUCCIÓN}

$\mathrm{E}$ l entorno laboral afecta la salud de los trabajadores, éste puede ser positivo y promoverla como también puede afectar negativamente causando padecimientos que vayan en detrimento de la calidad de vida. Al respecto la Organización Mundial de la Salud (OMS) y la Organización Panamericana de la Salud (OPS) (1). Declaran que el lugar de trabajo es el principal entorno que debe promover las condiciones que favorezcan la salud.

El personal de enfermería se caracteriza por desempeñarse en un ambiente de trabajo con altas exigencias ya que deben atender los requerimientos de los pacientes, las exigencias de otros trabajadores de la salud, aunado a que deben cumplir con horarios rotatorios con escaso tiempo para atender sus necesidades de descanso y alimentación. (2) Esto trae como consecuencia que la nutrición de éstos trabajadores se vea afectada presentando problemas de sobrepeso y obesidad.

Según datos de la OMS (1), las cifras de personas con sobrepeso y obesidad están en constante ascenso a nivel mundial para el año 2016 reportaron que más de 1900 millones de adultos de 18 o más años tenían sobrepeso, de los cuales, más de 650 millones eran obesos. El sobrepeso y la obesidad se definen como una acumulación anormal o excesiva de grasa que puede ser perjudicial para la salud. El índice de masa corporal (IMC) es un indicador simple de la relación entre el peso y la talla que se utiliza frecuentemente para identificar el sobrepeso y la obesidad en los adultos. La OMS define el sobrepeso: IMC igual o superior a 25 y la obesidad: IMC igual o superior a 30 . El IMC proporciona la medida más útil del sobrepeso y la obesidad en la población, pues es la misma para ambos sexos y para los adultos de todas las edades. Además de causar varias discapacidades físicas y problemas 
psicológicos un IMC elevado es un importante factor de riesgo para que una persona desarrolle una serie de Enfermedades No Transmisibles (ENT), las cuales fueron la principal causa de muerte en el 2012. Entre las ENT se encuentran los problemas cardiovasculares, el cáncer, la pancreatitis y la diabetes (3).

Desde este punto de vista la nutrición del personal de enfermería está en relación con el entorno laboral hospitalario donde se desempeñan. El objetivo de este trabajo es analizar el efecto del ambiente laboral en la nutrición del personal de enfermería y en consecuencia la identificación de factores que inciden en la aparición del sobrepeso y la obesidad. Con el propósito de comprender y explicar esta relación se ejecutó un meta análisis de investigaciones en las cuales se involucran estos dos eventos, el ambiente laboral hospitalario y su efecto sobre la nutrición del personal de enfermería.

Por la falta de tiempo y condiciones adecuadas en su entorno laboral el personal de enfermería tiende a presentar un patrón dietético negativo que se caracteriza por: (a) una baja ingesta de frutas, vegetales, cereales y fibra; (b) elevado consumo de azúcares simples (gaseosas y dulces); (c) alto porcentaje de grasas poliinsaturadas y grasas totales (d) deficiente ingesta de carbohidratos complejos, elevado consumo de productos refinados, comida rápida, chucherías y productos de bollería (4). Este patrón dietético conlleva a presentar exceso de peso y obesidad con las consecuencias asociadas a la salud.

En los entornos laborales, los empleados obesos presentan más fatiga, somnolencia y limitaciones físicas, lo que conlleva mayores riesgos de lesiones laborales que los empleados normopesos. (5). Un enfoque socio epidemiológico sugiere que ciertas características y condiciones de trabajo pueden causar comportamientos de salud negativos. (6). La tensión laboral se produce debido a las altas demandas psicológicas, combinadas con una baja latitud de control / decisión y la falta de apoyo social en el trabajo. En este contexto, la tensión laboral se muestra como un estrés laboral que puede afectar el comportamiento alimentario y la elección de alimentos, por ejemplo, una tendencia a comer alimentos más dulces y densos en energía (7).

En respuesta a los efectos negativos del sobrepeso y la obesidad la OMS ha creado el Plan de acción mundial para la prevención y el control de las Enfermedades No Transmisibles 20132020, que tiene por objeto cumplir los compromisos de la Declaración Política de las Naciones Unidas sobre las Enfermedades No Transmisibles (ENT). Este Plan recibió el respaldo de los Jefes de Estado y de Gobiernos en septiembre de 2011. El Plan de acción mundial contribuirá a realizar avances en nueve metas mundiales relativas a las ENT que deben alcanzarse no más tarde en el año 2025 , incluidas una reducción relativa del $25 \%$ en la mortalidad prematura a causa de dichas enfermedades para 2025 y una detención del aumento de la obesidad mundial para coincidir con las tasas de 2010 (1). Es importante conocer los factores que afectan la calidad nutricional para generar políticas de Estado y acciones efectivas para cumplir con las metas de mejor calidad de vida.

Uno de los factores que pueden influir en la salud del personal de enfermería, son los horarios extensos de trabajo causando 
tensión laboral o alterando los comportamientos de salud. El personal de enfermería es un pilar muy importante en el sector salud, por lo tanto su bienestar es garantía de un óptimo desempeño profesional. Se debe comprender que cualquier factor que incida de forma negativa en la salud del trabajador puede desencadenar deficiencias en el ejercicio de sus funciones y en el cuidado de los pacientes. (8).

El trabajo de enfermería se caracteriza por ser muy exigente, con largas jornadas laborales por turnos rotativos. Estas condiciones tienen efecto sobre la salud del trabajador pudiendo generar problemas de orden psicológico y físico. Uno de estos problemas es el sobrepeso, la obesidad y las ENT asociadas, que surgen como consecuencia de la calidad y cantidad de ingesta de alimentos. (8). Los horarios de trabajo adversos pueden conducir a la obesidad en el personal de enfermería; ya que el trabajo por turnos y las largas horas de trabajo interrumpen los tiempos normales de alimentación y reducen el acceso a alimentos saludables. (9-11) Debido a la falta de servicio de alimentos disponible, los trabajadores de horario nocturno tienden a comer alimentos poco saludables (por ejemplo, alto contenido de sal, azúcar) de máquinas expendedoras o alimentos pre envasado (12). Un estudio identificó que estos profesionales que cumplían el turno nocturno informaban que sus horarios de trabajo afectaban su vigor, la frecuencia de ejercicio, la vida social y familiar más que los que trabajaban en otros turnos (13). Sin embargo, son pocos los estudios que incorporan la relación entre obesidad y horarios de trabajo en el personal de enfermería. (14).
En las sociedades modernas, el trabajo nocturno ha sido reconocido como uno de los factores ocupacionales más prevalentes, afectando alrededor del 15\% al 20\% de la población activa en Europa y América del Norte (15), relacionándose con algunas enfermedades crónicas, incluidas las enfermedades cardiovasculares y el cáncer (16). A pesar de algunas inconsistencias, varios estudios transversales reportan asociaciones significativas entre el trabajo nocturno y el aumento del IMC. (17-18). Recientemente, Van Drongelen et al. (19) identificaron ocho estudios longitudinales que examinaron la relación entre el trabajo nocturno y el aumento de peso corporal, y concluyeron que la evidencia de una asociación cruda entre el trabajo por turnos y el aumento de peso corporal es fuerte pero se vuelve insuficiente después de controlar los factores de confusión. Desde la revisión de van Drongelen, solo se han publicado dos estudios longitudinales sobre este tema. Se encontró un mayor apoyo para la relación entre el trabajo nocturno y el aumento de peso para las mujeres, pero no para los hombres. $(20,21)$.

Si bien la mayoría de los hallazgos anteriores sobre las relaciones entre el trabajo nocturno y la obesidad fueron altamente sugerentes, se deben considerar varias limitaciones metodológicas. En particular, algunos de estos estudios $(17,18,22)$ se basaron en el peso y la estatura autoinformados, lo que pudo haber resultado en cierta imprecisión en la evaluación del IMC. Además, la mayoría de los estudios $(17,23,24,21)$ utilizaron información rigurosa sobre el sistema de trabajo realizado por el trabajador en el momento del estudio, por lo que las conclusiones sobre las relaciones causales 
fueron limitadas. Aparte de eso, los investigadores usualmente usaban el IMC para evaluar la composición corporal. Sin embargo, el IMC no distingue entre el sobrepeso debido al tejido muscular o grasa. Las medidas más precisas de la adiposidad abdominal, que estaban implicadas, por ejemplo, en trastornos metabólicos y cáncer de mama, rara vez se han considerado (25).

\section{METODOLOGÍA}

$\mathrm{S}$ e utilizó el diagrama de la declaración PRISMA (Preferred Reporting Items for Sistematycal Reviews and Meta-Analyses) (26) para realizar una revisión bibliográfica sistemática de investigaciones científicas con referencia al entorno laboral del personal de enfermería y sus aspectos nutricionales. Se escogió este diagrama ya que desde su publicación, se han reportado beneficios en la planificación, preparación y publicación de revisiones sistemáticas y meta-análisis. El diagrama se resume en cuatro etapas, la primera es la de Identificación de los estudios a través de la búsqueda en las bases de datos; la segunda es la etapa de cribado o selección, en esta etapa se eliminan los estudios duplicados y se excluyen aquellos que no presentan pertinencia con la investigación; la tercera es la de elegibilidad en la cual se incluyen todos los trabajos a texto completo para el análisis; por último la cuarta etapa constituida por los trabajos de revisión sistemática y metaanálisis.
En la etapa de búsqueda se utilizaron los siguientes aspectos: identificación del problema la cual se evidenció la descripción de la situación objeto de investigación, búsqueda sistemática de los documentos, procesamiento de información para el análisis, selección, evaluación y presentación de los datos. La búsqueda sistemática se realizó en seis bases de datos bibliográficas electrónicas, tales como: PubMed, Medline, SCOPUS, Wed of Science (Wos), CINAL y Cochrane. Se identificaron los artículos relacionados con los eventos de investigación, para lo cual se utilizó la pregunta Pico relacionada con ¿Cuál es la relación entre un entorno laboral y la nutrición del personal de enfermería del área hospitalaria? P: Personal de enfermería del área hospitalaria; I: Entorno laboral; C: Estado nutricional. La búsqueda sistemática inicio en septiembre del 2018, dando prioridad a estudios publicados a partir del año 2005. Los términos de búsqueda y $\mathrm{MeSH}$ incluyeron: "Estrés", "sobrepeso", "obesidad", "personal de enfermería", "práctica profesional", "estilo de vida", "estrés ocupacional" y "ambiente de trabajo". Por ejemplo utilizando la base de datos PubMed, se ingresó al buscador los términos: "stress and nursing and overweight" todo en idioma ingles y entre comillas. La Figura 1, muestra el diagrama de flujo PRISMA utilizado en la búsqueda y selección de los artículos de investigación relevantes. 


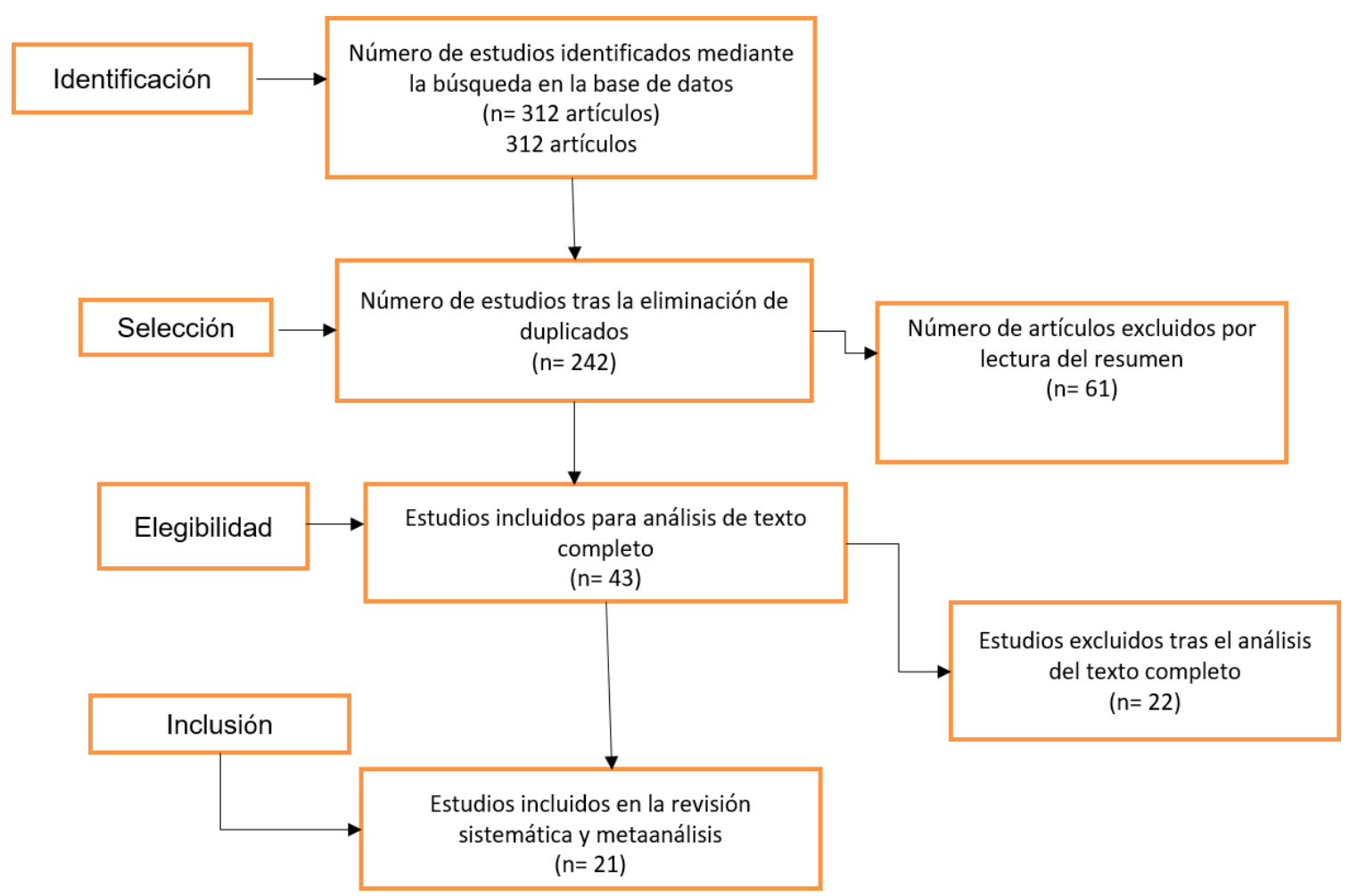

Figura 1. Diagrama de la declaración PRISMA (Preferred Reporting Items for Sistematycal Reviews and Meta-Analyses). Hutton Brian, Ferrán Catalá-López y David Moher, 2016.

La Identificación de investigaciones en todas las bases de datos quedó conformada por 312 artículos. Después de eliminar los artículos duplicados resultaron 242, se seleccionaron según el título de los artículos relevantes obteniendo 159, excluyendo de este grupo 61, después de un análisis y lectura rápida de resúmenes, de este grupo cuarenta y tres artículos fueron elegibles para lectura completa, de los cuales se excluyeron 22 debido a los años de publicación más recientes, quedando 21 artículos de los cuales dos correspondían a investigaciones cualitativas y diecisiete a estudios bajo el paradigma cuantitativo, estableciendo estos relevantes para la revisión.

Es importante mencionar que no hubo restricciones en el tipo de diseño del estudio, los artículos fueron seleccionados con base en los siguientes criterios de inclusión: 1. Todos son artículos de investigación son originales (por ejemplo, no revisiones sistemáticas, editoriales, tesis, resúmenes, etc.). 2. Publicaciones revisadas por pares de investigaciones originales que examinan el estrés laboral en relación con la carga de trabajo y las afecciones de salud en relación con el estado nutricional con efecto de sobrepeso y obesidad, se excluyeron artículos en los cuales no se relacionaban estas variables, se incluyeron aquellos que la investigación estaba enfocada al personal de enfermería en áreas hospitalarias y en los cuales se reflejaba niveles de estrés, IMC, sobrepeso y obesidad. 3. Los artículos recolectados se consideraron desde 2005 en adelante, 4. Clasificados en categoría de alto impacto JCR, 5. Escritos tanto en inglés como español. En esta revisión, se destacan investigaciones aplicadas al personal de 
enfermería enfermeras que laboran en áreas hospitalarias y específicamente a quienes trabajan turnos rotativos, el estudio converge en un factor desencadenante como el sobrepeso y obesidad.

La variable independiente utilizada en este estudio fue las alteraciones nutricionales en las enfermeras y enfermeros y las variables dependientes usadas fueron el estrés, sobrecarga y tensión laboral, calidad de dieta y conductas alimentarias, además, del entorno de práctica de enfermería, bajo el esquema correlacional de Pearson.

\section{RESULTADOS}

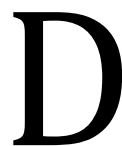

entro del proceso de revisión realizada en las cuales se apuntó este estudio estuvieron constituida por un conjunto de investigaciones relacionadas con la nutrición en el personal de enfermería como se muestran en la Tabla 1, en donde se analizaron los datos, tomando en consideración el tema, la metodología empleada, la población y muestra trabajada, como también de los instrumentos que fueron aplicados, con las finalidad de considerar los resultados más representativos dentro del desarrollo de cada investigación, su origen, procedencia o contexto de estudio, y por último el nivel académico científico y la relevancia del estudio con relación a las alteraciones nutricionales en el personal de enfermería. 
Tabla 1. Análisis sistemático relacionado con alteraciones nutricionales del personal de enfermería

TEMA METODOLOGÍA EMPLEADA RESULTADOS CLAVES

Factores ocupacionales asociados con la obesidad y la actividad física en el tiempo libre entre las enfermeras

Dal Lae Chin, Soohyun Namb, SooJeong Lee (2016)

EEUU-California

\section{Fue Estudio transversal.}

Muestra de $\mathrm{N}=394$ enfermeras, seleccionadas al azar de la lista de la Junta de Enfermería Registrada de California.

Instrumentos: *IMC (CDC 2012).

*Physical Activity

Guidelines for Americans (USDHHS, 2008).

*Physical activity 2 or more days a week

(USDHHS, 2008).

*Physical Workload

Index Questionnaire (PWIQ).

*Data were analyzed using SPSS version 20

(SPSS, Chicago, IL)
De los participantes, el $31,1 \%$ tenía sobrepeso y el $17,6 \%$ era obeso.

Para la actividad física, el $41.3 \%$ realizó actividad física aeróbica regular y el 56.6\% realizó actividades de fortalecimiento muscular 2 o más días a la semana.

- Se encontraron asociaciones significativas entre el IMC y la actividad física: la proporción de enfermeras obesas fue significativamente mayor entre las enfermeras que no participaron en la actividad física aeróbica regular (23.7\% vs. $9.5 \%$, p = .004) y en la actividad regular de fortalecimiento muscular $(23.6 \%$ contra $13.4 \%, \mathrm{p}=.040)$, en comparación con las enfermeras que realizan actividad física regular.

- Ningún factor sociodemográfico se asoció significativamente con la actividad física regular, pero la proporción de actividad física regular de fortalecimiento muscular tendió a disminuir con el aumento de la edad $(\mathrm{p}=$ .084).

- -La prevalencia de sobrepeso / obesidad fue significativamente mayor entre las enfermeras que trabajaron a tiempo completo en comparación con las enfermeras a tiempo parcial o por día (52.1\% vs. $37.8 \%, p=.015)$ y entre las enfermeras que trabajaron $40 \mathrm{~h}$ por semana en comparación para aquellos que trabajaron $<40 \mathrm{~h}$ por semana $(58.9 \%$ vs. $39.9 \%$, p <.001)

- Todas las variables significativas en el análisis bivariado mantuvieron asociaciones significativas en el análisis multivariable, controlando la edad, el género, la raza / etnia, la educación y el dolor musculoesquelético. Además, el título del trabajo y el turno de trabajo mostraron asociaciones significativas con sobrepeso / obesidad o actividad física regular de fortalecimiento muscular en el análisis multivariable.

- En particular, las enfermeras que trabajaron en turnos nocturnos tuvieron significativamente menos probabilidades de realizar actividad física regular para fortalecer los músculos (OR = 0,44; IC del 95\%: 0,25-0,77) y tendían a ser menos propensas a realizar actividad física aeróbica $(\mathrm{OR}=$ $0,59)$. 
Asociación del índice de masa corporal con el estilo de vida y el trabajo por turnos rotativos en

enfermeras japonesas

Yuki Tada, Yukari Kawano, Izumi Maeda, Takahiro Yoshizaki, Ayaka Sunami, Yuri Yokoyama, Harumi

Matsumoto,

Azumi Hida1, Taiki Komatsu, and

Fumiharu Togo. Kanagawa (2014) Japón

\section{Estudio transversa}

Muestra Enfermeras japonesas $\mathrm{N}=1179$

jornaleros y 1579 turnos rotativos, de 20 a 59 años de edad.

\section{Instrumentos}

* Excel Eiyoukun Food Frequency

Questionnaires.

* Los hábitos de consumo de alcohol y tabaco

se evaluaron según las definiciones de la

encuesta nacional de salud y nutrición.

* Cuestionario Internacional de Actividad

Física.
- $\quad$ EI IMC fue significativamente mayor $(P<0.05)$ en SW (Trabajadores de turnos rotativos) que en DW (Trabajadores diurnos).

- La frecuencia de los sujetos que estaban en el grupo de obesos fue significativamente mayor $(P<0.05)$ en SW que en DW.

- Las duraciones del sueño en las noches entre días en el turno de día y las noches entre días de descanso fueron significativamente más cortas (P $<0.05$ ) y más largas, respectivamente, en SW en comparación con DW.

- $\quad$-El nivel de actividad física no difirió entre los grupos.

- La duración corta del sueño podría estar asociada con una reducción de la leptina y un aumento de la ingesta total de energía y grasa, aunque la ingesta total de energía no difirió entre el DW y el SW.

- Otros factores, como el aumento de la hormona lipogénica cortisol y la activación de las vías inflamatorias pueden explicar la asociación entre la duración del sueño y el IMC. 
Asociación de trabajo nocturno rotativo con IMC y obesidad abdominal en enfermeras y parteras

Beata Peplonska, Agnieszka Bukowska, Wojciech Sobala Polonia(2015)

\section{Estudio transversal}

\section{Muestra:}

$\mathrm{N}=724$ enfermeras y matronas, con edades comprendidas entre 40 y 60 años (354 turnos nocturnos rotativos y 370 trabajadoras diurnas)

\section{Instrumentos:}

${ }^{*}$ Cuestionario Internacional de Actividad Física (IPAQ).

${ }^{*}$ Cuestionario de Calidad del Sueño de Pittsburgh

*Antropometría autoinformada y medida Se les preguntó a las mujeres sobre su peso y altura actuales, y tuvieron que indicar su imagen de forma corporal (actual y a los 20 años) utilizando una escala de silueta de 9 niveles.
- Obesidad (IMC? $30 \mathrm{~kg} / \mathrm{m} 2$ ) y obesidad abdominal como se indica por $\mathrm{WHR}>0.85$ y WHtR> 0.55 (0.6) fueron ligeramente más frecuentes entre las mujeres que actualmente trabajan en turnos nocturnos rotativos, pero las diferencias no fueron estadísticamente significativas.

- Asociaciones positivas y estadísticamente significativas para la exposición laboral acumulada en el turno nocturno expresada como el número total de turnos nocturnos (o de turnos nocturnos) e IMC, WC, HC y WHtR, con un IMC que aumenta en $0.477 \mathrm{~kg} / \mathrm{m} 2$ por cada 1000 turnos nocturnos y de $0,432 \mathrm{~kg} / \mathrm{m} 2$ por 10000 horas de turno nocturno, el WC aumenta respectivamente en $1,089 \mathrm{~cm}$ y $0,997 \mathrm{~cm}$, y HC en $0,72 \mathrm{~cm}$, y WHtR 0,007.

- El trabajo nocturno, tanto actual como acumulativo, se asoció sistemáticamente con la obesidad (IMC? $30 \mathrm{~kg} / \mathrm{m} 2$ ), con un OR de 3.9 (IC 95\%: 1.59 .9 ) en mujeres que informaron ocho turnos nocturnos o más por mes.

- Una mayor frecuencia de turnos nocturnos también se asoció significativamente con la obesidad abdominal, con OR $=2.4$ (IC 95\%:1.24.5) para $\mathrm{WC}>88 \mathrm{~cm}, \mathrm{OR}=2.8$

- $\quad(95 \% \mathrm{Cl}: 1.3-6.0)$ para HC? $108 \mathrm{~cm}, \mathrm{OR}=2.4(95 \% \mathrm{Cl}: 1.2-4.9)$ para $\mathrm{WHR}>0.85$ y $\mathrm{OR}=2.7(95 \% \mathrm{Cl}: 1.3-5.6)$ para $\mathrm{WHtR}>0.55(0.6)$.

- También observamos tendencias positivas significativas para la asociación entre las horas acumuladas de trabajo nocturno y el IMC> $30 \mathrm{~kg} / \mathrm{m} 2, \mathrm{WC}>$ $88 \mathrm{~cm}$, y HC? $102.5 \mathrm{~cm}-108 \mathrm{~cm}$, así como el número de turnos nocturnos acumulados y WHtR> 0.55 (0.6).

- Todas las asociaciones restantes fueron positivas y en la dirección esperada, aunque no fueron compatibles con pruebas estadísticamente significativas.

- Clasificación de los sujetos según las categorías de IMC de la OMS, utilizando el IMC calculado a partir de mediciones físicas y clases de IMC basadas en la imagen de la forma del cuerpo actual auto percibida. La correlación entre las dos categorizaciones fue satisfactoria (coeficiente de correlación de Pearson 0,81). 


\section{TEMA}

Conductas de autocuidado de las enfermeras relacionadas con el peso y el estrés.

Eun-Shim Nahm, PhD, RN, FAANa Joan Warren, PhD, RN-BC, NEA$\mathrm{BCb}$,

Shijun Zhu, PhDa, Minjeong An,

MSN, RN, FNP-BCa, Jeanine Brown,

MS, RN, CCRCa EE.UU.(2012)

\section{METODOLOGÍA EMPLEADA}

\section{Estudio transversa}

\section{Muestra: $\mathrm{N}=169$ enfermeras}

\section{Instrumentos:}

* El índice de masa corporal (IMC) se calculó utilizando la altura y el peso autoinformados.

*Preguntas específicas sobre el patrón de dieta.

*Escala para valorar estrés PSS (Cohen, Kamarck y Mermelstein, 1983; Cohen y Williamson, 1988.

\section{RESULTADOS CLAVES}

- Un total de 59.2\% (n $1 / 4$ 100) tenía sobrepeso, entre los cuales aproximadamente la mitad eran obesos ( $n$ 1/4 53)

- La mayoría de los participantes (n $1 / 4122,72.2 \%$ ) eran enfermeras clínicas, y $10.0 \%$ (n $1 / 417)$ fueron administradores o gerentes.

- $\quad$-Más de la mitad de los participantes ( $n=91,53.8 \%$ ) informaron que no comían comidas regulares.

- Un total de 41 participantes informaron que a menudo estaban demasiado ocupados para tomar descansos para comer mientras trabajaban. Diecinueve participantes citaron la vida ocupada en general, y 17 citaron dificultades relacionadas con el trabajo por turnos (por ejemplo, turno nocturno y turno de 12 horas).

- La mayoría de los participantes (n 1/4 122, 72.2\%) sintieron que no estaban haciendo suficiente ejercicio.

- El promedio de minutos por semana para las actividades de nivel de vigor (p. Ej., Trotar, aeróbicos) fue de 42.41+- 80.3 (n 1/4 135) y el ejercicio de nivel moderado (p. Ej., Andar en bicicleta, caminar) fue de 113+- 118.2. El promedio total de minutos por semana por ejercicio fue de $97+-116.9$.

- $\quad$ El nivel de estrés medio evaluado por el PSS de 4 ítems fue de $6.49+-3.19$ (rango 0-16). El enfoque más utilizado fue comer (n 1/4 32), seguido de ejercicio $(n 1 / 431)$, el uso de técnicas de relajación (n 1/4 27) y hablar con alguien ( $\left.n^{1 / 4} 26\right)$.

- Los participantes que tenían horarios de comidas regulares y hacían más ejercicio tenían puntuaciones de IMC más bajas.

- El nivel de estrés y la edad no se asociaron significativamente con el IMC Esto podría estar relacionado con el hecho de que la mayoría de las enfermeras ( $\mathrm{n} 1 / 4116,68.3 \%$ ) tenían 45 años o más. Los participantes con mayor estrés tuvieron patrones de comida más irregulares $(r \quad 1 / 4.32, P$ $<0.001)$. 
Asociación de conductas

alimentarias con preferencia diurna y turnos rotativos en enfermeras

japonesas

Takahiro Yoshizaki, Yukari Kawano,

Osamu Noguchi, Junko Onishi,

Reiko Teramoto, Ayaka Sunami, Yuri

Yokoyama, Yuki Tada, Azumi Hida,

Fumiharu Togo. Ome Tokio

\section{Estudio transversal}

Muestra: $\mathrm{N}=162$ (39 trabajadores por día y 123 trabajadores de turnos rotatorios, con edades entre 21-63 años)

\section{Instrumentos:}

* Versión japonesa del cuestionario ME por Torsvall y Akerstedt se usó para medir la preferencia selectiva por la actividad en la mañana o en la noche.

* Cuestionario sobre conductas alimentarias emitido por la Sociedad de Japón para el Estudio de la Obesidad
La duración media total de la experiencia en su trabajo actual fue de $122 \pm 10.3$

(SD) años. En este estudio, los individuos que trabajaron solo en turnos diurnos fijos (es decir, 08: 30-17: 15 horas) se definieron como "trabajadores diurnos", mientras que los que trabajaron en un sistema de dos turnos (días y noches, a las 08: 3017:15 y 16: 30- 09:15.

El estado civil y el estado residencial se asociaron significativamente con el programa de trabajo actual $(p<0.05)$.

- La puntuación ME para los trabajadores de turnos rotativos fue significativamente más bajo en comparación con los trabajadores diurnos ( $\mathrm{p}<0.05$ ), lo que indica una mayor actividad nocturna / menos trabajo matutino entre los trabajadores de turnos rotativos en comparación con los trabajadores diurnos.

- Los puntajes para los contenidos de comidas y los patrones de alimentación temporales difirieron significativamente entre los grupos ( $p$ $<0.05$ ), lo que indica una dieta desequilibrada y un tiempo más irregular de Comidas entre los trabajadores de turnos rotativos en comparación con los jornaleros. Las puntuaciones para otros comportamientos alimenticios no difirieron entre los grupos ( $p>0.05$ ).

- $\quad$ La regresión lineal simple mostró que el trabajo por turnos rotativos y una puntuación ME más baja se asociaron significativamente $(p<0.05)$ con puntuaciones más altas para los contenidos de comidas y los patrones de alimentación temporal.

- La regresión lineal multivariable mostró que la puntuación ME fue significativamente $(\mathrm{p}<0.05$ ) asociada con la puntuación para los contenidos de comida, mientras que el efecto del trabajo de turno rotatorio no fue $(p=0.245)$

- $\quad{ }^{*}$ Con respecto a los patrones de alimentación temporal, la puntuación ME se asoció significativamente con la puntuación $(p<0,05)$, mientras que el trabajo por turnos rotativos se atenuó solo hasta un nivel de tendencia $(\mathrm{p}=$ $0,060)$.

- Las variables de las características demográficas $(p<0.05)$ diferían significativamente entre los grupos (es decir, edad, años de experiencia como trabajador de turno rotativo, estado civil, estado residencial y número de turnos nocturnos durante el año). 


\section{TEMA}

METODOLOGÍA EMPLEADA

\section{RESULTADOS CLAVES}

Estrés laboral y horarios de trabajo en relación con la obesidad de las enfermeras.

Kihye Han, $\mathrm{PhD}, \mathrm{RN}$

Alison M. Trinkoff, ScD, RN

Carla L. Storr, ScD

Jeanne Geiger-Brown, PhD, RN

E.E.U.U

Illinois y Carolina del Norte (2011)

Estudio transversal

Muestra: $\mathrm{N}=2,103$ enfermeras

Instrumentos: * Cuestionario de contenido (demanda-control y apoyo en el trabajo) JCQ y el NWI-R. (escala de entorno practica de enfermería) NWHS.

*La obesidad se basó en un índice de masa corporal derivado (IMC), dividiendo el peso en kilogramos por el cuadrado de la altura en metros, que luego se clasificó en 2 grupos de peso: bajo peso / normal (UW / NW; IMC G $25,0 \mathrm{~kg} / \mathrm{m} 2)$ y OW / OB (IMC Q $25.0 \mathrm{~kg} /$ $\mathrm{m} 2$
- La prevalencia de sobrepeso (IMC Q $25.0 \mathrm{~kg} / \mathrm{m} 2$ ) para la muestra fue de $55.0 \%$, y el $27.1 \%$ de la muestra era obesa (IMC Q $30.0 \mathrm{~kg} / \mathrm{m} 2$ ). No hubo variación en estas prevalencias según el lugar de empleo (hospital versus no hospitalario).

- Cuando se compararon con las enfermeras de UW / NW, las enfermeras de OW / OB eran aproximadamente 2.5 años más antiguas (PG .01), más probabilidades de ser afroamericanas (PG 01) con menos educación (PG .01), trabajó como RN más tiempo (PG .01), y es más probable que trabaje a tiempo completo $(\mathrm{P}=.02 ;)$.

- Además, las enfermeras de OW / OB informaron significativamente más síntomas depresivos (PG .01), percibieron que su salud era peor (PG .01), realizaron menos ejercicio $(P G .01)$ y tuvieron un sueño más inquieto $(P=$ .03) que las enfermeras de UW / NW hicieron, mientras que la cantidad de sueño no difirió entre los 2 grupos $(P=.40)$. 


\section{TEMA}

Demografía y factores psicológicos asociados con la adiposidad en enfermeras

Bernarda Sánchez-Jiménez, Reyna Sámano, Daniela Chinchilla-Ochoa, Rosa Morales-Hernández and

México (2018)

\section{METODOLOGÍA EMPLEADA}

Estudio transversal

\section{Muestra: $\mathrm{N}=265$ enfermeras}

\section{Instrumentos:}

*EI IMC se calculó como el peso dividido por la altura al cuadrado (kg / m2) y Ow / Ob se clasificaron de acuerdo con los puntos de corte de la Organización Mundial de la Salud. Ow fue definido como BMI? 25 y Ob? 30.

*La edad, la antigüedad laboral, el área de servicio, el turno, el estado civil y el número de niños se determinaron mediante un cuestionario interno validado.

* Se consideró la hiperglucemia si la glucosa en ayunas era de $100 \mathrm{mg} / \mathrm{dL}$. El síndrome metabólico se definió de acuerdo con el Programa Nacional de Educación sobre el Colesterol y el Panel de Tratamiento para Terceros Adultos (NCEP-ATPIII) con tres o más de las siguientes características: glucosa plasmática ¿en ayunas? ¿100 mg / dL, presión arterial? ¿130 /? ¿85 $\mathrm{mmHg}$, triglicéridos? ¿150 mg / dL, $\mathrm{HDL}<50 \mathrm{mg} / \mathrm{dL}$ y / o cintura? $88 \mathrm{~cm}$.

*Autoestima, prueba de Goldberg

* IBM SPSS Statistics versión 22.

\section{RESULTADOS CLAVES}

- $\quad$ La adiposidad estuvo presente en $79.6 \%$ : Ow en $43.4 \%$ y Ob en $36.2 \%$;

El $68 \%$ tenía circunferencia de cintura> $80 \mathrm{~cm} ; 30 \%>88 \mathrm{~cm}$; El $65 \%$ y el $78 \%$ tenían $>0,80$ y 0,50 índice de cintura / cadera, respectivamente.

- Las enfermeras del turno nocturno tuvieron el porcentaje más alto de ABCD (87\%) en comparación con los enfermeros de los turnos matutinos $(80 \%)$ o vespertinos $(67 \%)(p=0,031)$.

- $\quad A B C D$ se asoció con la antigüedad en el trabajo (+- 15 años) $(p=0.006)$, pero no con la edad.

- Solo una enfermera tenía hipertensión, el 25\% tenía síndrome metabólico y el $67,5 \%$ tenía glucosa en ayunas anormal.

- El $45 \%$ y el $41 \%$ tenían hipercolesterolemia e hipertrigliceridemia, respectivamente. Hiperglucemia $(p=0.05)$, hipercolesterolemia $(p=0.026)$ e hipertrigliceridemia ( $p$ ? 0.001) se asociaron con ABCD.

- Acerca de los factores de estilo de vida explorados, el $42 \%$ practicó ejercicio, pero solo el 3\% siguió las recomendaciones internacionales: $2.5 \mathrm{~h}$ por semana y 30 min en cada sesión. Las enfermeras que practicaron actividad física menos de tres días por semana, tuvieron riesgo de tener ABCD $(O R=4.1)$ y menos de 30 min en cada sesión también tuvieron riesgo $(\mathrm{OR}=2)$.

- Por otro lado, sobre el estado emocional, el 26\% mostró una baja autoestima y un $10 \%$ de angustia emocional. Estas condiciones no se asociaron con $A B C D$, pero se asoció con baja autoestima (OR $=2, I C 95 \%$ $1.150-3.07, p=0.023$ ) y angustia emocional (OR $=4$, IC 95\% $1.472-$ $13.078, p=0.012$ ) con un estilo de vida poco saludable (menos de 3 días por semana y / o menos de 30 minutos por sesión de actividad física y malos hábitos alimenticios. 


\section{TEMA}

Comportamientos de salud y

sobrepeso en empleados de asilos de ancianos: contribución de los factores de estrés en el lugar de trabajo e implicaciones para la promoción de la salud en el lugar de trabajo.

Helena Miranda, Rebecca J. Gore, Jon Boyer, Suzanne Nobrega, and Laura Punnett. Varios estados de U.S (2015)

Prevalencia del sobrepeso y la obesidad entre las enfermeras en Escocia.

Richard G. Kyle, Rosie A. Neall, lain M. Atherton. Escocia

\section{METODOLOGÍA EMPLEADA}

Estudio transversal

Muestra: N=1506 personal de salud hogares de ancianos. Instrumentos:

*Job Content Questionnaire (JCQ).

*El índice de masa corporal (IMC) se calculó a partir del peso y la talla informados por el paciente. "Obeso" se definió como un IMC de 30.0 o superior

Estudio transversal Muestra: $\mathrm{N}=13,483$ adultos: clasificados en cuatro grupos ocupacionales: enfermeras $(n=411)$, otros profesionales de la salud $(n=320)$, personal de atención no calificado $(n=685)$ y Personas empleadas en ocupaciones no relacionadas con la salud $(n=12,067)$

Instrumentos:

* Scottish Health Survey. ( utiliza para estimar la prevalencia de afecciones de salud, factores de riesgo de enfermedad, realiza un seguimiento de las tendencias de salud) Encusta de salud Escocesa.

* Los entrevistadores midieron la altura y el peso de los participantes a partir de los cuales se derivó el IMC (Scottish Center for Social Research, 2008). Siguiendo la clasificación de la Organización Mundial de la Salud (OMS), el IMC se clasificó la Organización Mundial de la Salud (OMS), el IMC se clasificó 'sobrepeso' (IMC = 25.0-29.9) y 'obeso' (IMC ? 30)

* Clasificación profesional estándar SOC2000 (para los años de encuesta 2008-2011) y SOC2010 (2012).

\section{RESULTADOS CLAVES}

Los encuestados eran 34\% obesos, $24 \%$ fuman actualmente y $23 \%$ físicamente inactivos fuera del trabajo.

- Uno de cada cinco tenía al menos otro trabajo remunerado. -Los empleados reportaron altas demandas psicológicas en el trabajo (88\% de los encuestados), incómodas posturas (65\%), mal clima de seguridad (60\%), levantamiento de cargas pesadas (57\%) y desequilibrio entre el trabajo y la familia $(43 \%)$.

- Los trabajadores menores de 40 años reportaron sistemáticamente más factores de estrés en el lugar de trabajo que aquellos mayores de 40 años especialmente la carga de trabajo física y los problemas de seguridad.

- El riesgo de obesidad se asoció linealmente a los modelos multivariables con la suma de estas características ocupacionales: baja actitud de decisión, bajo apoyo de compañeros de trabajo, levantamiento de cargas pesadas, trabajo nocturno y asalto físico reciente.

13,483 personas en el conjunto de datos final para su análisis. La muestra incluyó $411(3,0 \%)$ enfermeras, $320(2,2 \%)$ otros profesionales de la salud, $685(5,1 \%)$ trabajadores de atención y $12,067(89,5 \%)$ en ocupaciones no relacionadas con la salud.

- La prevalencia de sobrepeso y obesidad fue mayor en las enfermeras que en otras profesionales de la salud $(51,3 \%$, IC $95 \%, 45,8,56,7)$, aunque más de la mitad de ese grupo de comparación, sin embargo, tenían un IMC que indicaba que estaban por encima de un peso saludable.

Un modelo de regresión logística ajustado por la composición sociodemográfica indicó que, en comparación con las enfermeras, las probabilidades de tener sobrepeso u obesidad fueron estadísticamente significativamente más bajas para otros profesionales de la salud (Odds Ratio [OR] 0,45, IC 0,33, $0,61)$ y en personas no relacionadas con la salud. Ocupaciones (OR 0.78, IC 0.62, 0.97) 
TEMA

Asociaciones entre la salud autoevaluada y las condiciones psicosociales,

Factores de estilo de vida y recursos

de salud entre enfermeras de hospitales.

Vilija Malinauskiene, Palmira Leisyte, Romualdas Malinauskas \& Kristina Kirtiklyte. Lithuania (2011)

\section{METODOLOGÍA EMPLEADA}

\section{Estudio Transversal Muestra: $\mathrm{N}=748$} enfermeras.

\section{Instrumentos:}

*Se utilizó el Cuestionario de salud general (GHQ-12) para medir el nivel de dificultad mental; cubre sentimientos de tensión, depresión, incapacidad para sobrellevar la situación, insomnio basado en la ansiedad, falta de confianza y estima y otros síntomas de malestar mental.

* Las características psicosociales del trabajo se midieron utilizando la versión sueca del cuestionario Karasek Demand-Control.

* El consumo de tabaco se clasificó como no fumador, fumador y ex fumador. Aquellos que habían dejado de fumar <2 años antes de la inclusión en el estudio se consideraron fumadores actuales.

*El índice de masa corporal, definido como el peso en kilogramos dividido por la altura en metros cuadrados, se clasificó como peso normal (IMC, 18Æ5-24Æ9 kg/m2), sobrepeso (IMC, 25Æ0-29/E9 kg / m2) y obesidad (IMC $\ddagger 30$ Е0 kg / m2).

${ }^{*}$ El consumo de alcohol se evaluó en una escala de 6 puntos que va desde "nunca" hasta "diario".

* The SPSS versión 13.0

\section{RESULTADOS CLAVES}

- Las enfermeras que calificaron su salud como "buena" o "muy buena" fueron bastante bajas en nuestro estudio (39.6\%); de hecho, el $58.3 \%$ de las enfermeras evaluaron su salud como "justa" y el $2.1 \%$ como "pobres" y "muy pobres".

- Resultados del análisis de regresión logística. Para la salud autoevaluada. Importantes OR ajustadas por edad de altas demandas laborales, bajo control laboral, bajo apoyo social, insatisfacción laboral, exposición a la intimidación durante más de 1 año, eventos potencialmente mortales, consumo de alcohol, baja actividad física, obesidad, bajo sentido de coherencia y malestar mental.

- Las asociaciones entre la salud autoevaluada negativa aumentaron dramáticamente con la edad (OR = 15/E59; IC del 95\%: 6-80-35-70) para las personas de 55 a 70 años en comparación con las de 24 a 34 años y fueron las más fuertes con altas demandas de trabajo, baja control del trabajo, bajo apoyo social en el trabajo, falta de tiempo libre para actividades físicas y eventos que amenazan la vida.

- Encontramos asociaciones constantes entre la angustia mental (medida por GHQ-12) y la salud autoevaluada (OR = 2ÆE8; 95\% Cl 1Æ65-4Æ75) y entre la angustia mental y el estrés laboral (correlación de Pearson 0 E230, $\mathrm{P}<0$ ÆE01)

- $\quad$ IMC normal 295 enfermeras, sobrepeso 300 y obesidad 153. 


\section{TEMA}

Un análisis transversal de la asociación entre trabajo nocturno o turno rotativo y sobrepeso / obesidad entre enfermeras y parteras.

Isabella Zhao, RN, BN, BN (Hons I), Fiona Bogossian, RN, DipAppSci, BAppSci, MPH, PhD, MACMI and Catherine Turner, RN, BA, Grad Dip, Ed, MN, PhD

Australia, Nueva Zelanda y el Reino Unido (2012)

\section{METODOLOGÍA EMPLEADA}

\section{RESULTADOS CLAVES}

Estudio Transversal muestra: $\mathrm{N}=7604$ enfermeras y parteras.

\section{Instrumentos:}

*De acuerdo con los estándares de clasificación de IMC de la Organización

Mundial de la Salud, 33 un IMC normal y saludable varía de 18.5 a 24.9. El sobrepeso y la obesidad se refieren a un IMC de 25.0 29.9 y 30.0 o más, respectivamente.

* La calidad de la dieta se midió utilizando el puntaje australiano de alimentos recomendados (ARFS) 74 ítems en el que cada ítem tiene un formato de respuesta de "sí / no".

*La actividad física fue evaluada por el Cuestionario Internacional de Actividad Física (IPAQ).

(IPAQ). artículos de Nurses 'Health Studies (NHS).

*El SF-36 se puede dividir en dos medidas de resumen agregadas del componente físico (puntaje del componente físico [PCS]) y de componente mental (puntaje del componente mental [MCS]), 56 que reflejan la salud física mental [MCS]), 56 que reflejan la salud física general percibida y la salud mental gen ${ }^{\star}$ El estado menopáusico se obtuvo preguntando a los participantes si sus forma permanente.
En nuestra muestra de estudio, 2086 enfermeras y matronas tenían entre 21 y 69 años $(43.4+9.6)$ años.

- Entre los tres grupos de exposición, los trabajadores por turnos rotativos eran casi 5 años más jóvenes que los trabajadores de turno diurno y nocturno (40.5 \pm 10.2 vs $45.2 \pm 8.7$ y $45.3 \pm 9.0$ años, respectivamente, $p<0.001$ ).

De los participantes, 1212 eran trabajadores diurnos, 759 trabajaban turnos rotativos y 115 trabajaban turnos nocturnos. -En esta muestra, casi el $60 \%$ de los participantes fueron clasificados como con sobrepeso $(31,7 \%)$ u obesos $(27,1 \%)$.

El mayor porcentaje de sobrepeso se encontró en los trabajadores con turnos rotativos (33.3\%), mientras que el porcentaje más alto de obesidad se encontró en los trabajadores de turno nocturno (31.3\%).

Con respecto a los factores de estilo de vida modificables, la mayoría de los participantes informó tener una dieta de alta calidad (61.5\%) y un alto nivel de actividad física $(51.7 \%)$

La proporción de participantes que informaron una alta calidad de la dieta fue mayor en los trabajadores de turno nocturno $(67.8 \%)$ que en los otros dos grupos.

-El grupo con el porcentaje más alto que reportó un alto nivel de actividad física fue la rotación de trabajadores por turnos $(59.7 \%)$, seguida por los trabajadores de turno nocturno (53\%). - La mayoría de los participantes (56\%) informaron que nunca habían fumado un cigarrillo, el $11.7 \%$ eran fumadores actuales y el $32.4 \%$ eran ex fumadores. Los trabajadores de turno nocturno tuvieron la mayor proporción de fumadores actuales (16.5\%).

Tres cuartas partes de los participantes se identificaron como bebedores de bajo riesgo y aproximadamente el $10 \%$ de ellos eran bebedores arriesgados o de alto riesgo.

- -Las proporciones de mujeres menopáusicas fueron bastante similares entre las trabajadoras de turno diurno y nocturno $(68.8 \%$ frente a $66.1 \%)$, se encontró un porcentaje mucho mayor de mujeres menopáusicas en las trabajadoras de turno rotatorio.

- Tanto los componentes físicos como mentales del SF-36 se encontraban dentro del rango esperado (20 a 58 para PCS y 17 a 62 para MCS), 61 y fueron bastante similares en los tres grupos. 


\section{TEMA}

Comparación del estrés laboral y la obesidad en enfermeras con

pacientes favorables y horarios de

trabajo desfavorables.

Kihye Han, PhD, Alison M. Trinkoff, ScD, Carla L. Storr, ScD, Jeanne Geiger-Brown, PhD,

Karen L. Johnson, PhD, and Sungae

Park, PhD.

Illinois y Carolina del Norte (2012)

\section{METODOLOGÍA EMPLEADA}

Estudio Transversal muestra: $N=1724$ nurses.

Instrumentos: *Encuesta de enfermeras de la línea de base de Nurses Worklife and Health Study III.

*Modelo Karasek de la tensión laboral.

*Las estimaciones del índice de masa

corporal se dividieron luego en dos grupos:

bajo peso / peso normal (UW / NW; $<25.0 \mathrm{~kg} /$ m2) y sobrepeso / obesidad (OW / OB; $\geq 25.0$ $\mathrm{kg} / \mathrm{m} 2)$

\section{RESULTADOS CLAVES}

- En comparación con las enfermeras con SS favorables, las enfermeras con PS desfavorables fueron significativamente más jóvenes (edad media, 44 vs. 46 años; $P<0.01$ ), tuvieron menos educación ( $47 \%$ vs $57 \%$ con una licenciatura 0 más; $P<0.01$ ), tuvieron menos años de experiencia en enfermería registrada (media 18 vs. 20 años; $P<0.01$ ), y eran más propensos a trabajar en hospitales ( $63 \%$ vs $55 \%$ trabajando en hospitales; $P<0.01)$.

- Las enfermeras con TS desfavorables durmieron menos $(P<0.01)$ y tuvieron más sueño inquieto $(P<0.01)$ que las que tuvieron WS favorables, mientras que la proporción de OW / OB no fue diferente.

- Entre las enfermeras con SS favorables, las enfermeras con OW / OB informaron significativamente más conductas poco saludables (es decir, fumar y consumir alcohol).

- Las enfermeras con sobrepeso / obesidad también informaron significativamente mayores aportes de enfermeras en sus puestos de trabajo (OR, $1.21 ; 95 \% \mathrm{Cl}, 1.02-1.44)$ y un jefe menos solidario en el trabajo (OR, 0.83; $95 \% \mathrm{Cl}, 0.68-0.99$ ) en comparación con las enfermeras que fueron UW / NW.

- Por el contrario, entre las enfermeras con WW desfavorables, las enfermeras con OW / OB tuvieron significativamente menos probabilidades de practicar conductas saludables (es decir, el ejercicio y el sueño) que las enfermeras con UW / NW (OR, 0.79; 95\% Cl, 0.66-0.95).

- Ningún componente de estrés laboral se relacionó significativamente con ser OW/OB entre aquellos con WS desfavorables. 


\section{TEMA}

Sobrepeso y obesidad en

enfermeras, enfermeras de práctica

avanzada y enfermeras educadoras

Sally K. Miller, PhD, Patricia T.

Alpert, DrPH, and, Chad L. Cross,

PhD. Noreste, la costa este, medio

oeste, sur, suroeste y oeste de los

EE. UU (2007)

\section{METODOLOGÍA EMPLEADA}

Estudio Transversal Muestra: $\mathrm{N}=760$ enfermeras

Instrumentos: *Encuesta salud y nutrición (NHANES).

${ }^{*}$ De acuerdo con los criterios del Grupo de trabajo internacional sobre la obesidad, e sobrepeso se define clínicamente como un IMC> 25 y la obesidad como un IMC> 30 (Flegal, Carroll, Kuczmarski y Johnson, 1998).

\section{RESULTADOS CLAVES}

- $\quad$ El IMC promedio de los encuestados varió de 25.9 a 29.5, lo que indica que los seis estados informaron un IMC promedio en el rango de sobrepeso.

- El noventa y dos por ciento de los encuestados eran mujeres. Las edades oscilaron entre 22 y 85 años $(M=50.2, S D=11.88$, mediana $=51.0)$, con más del $50 \%$ entre las edades de 45 y 64 años.

- El índice de masa corporal media grande (IMC) de las enfermeras encuestadas fue de 27.2. Casi el 54\% tenía sobrepeso u obesidad. Cincuenta y tres por ciento de estas enfermeras informan que tienen sobrepeso pero que no tienen la motivación para hacer cambios en el estilo de vida. El cuarenta por ciento no puede perder peso a pesar de una dieta saludable y hábitos de ejercicio.

- Solo el $26 \%$ de los encuestados usa el IMC para hacer juicios clínicos de sobrepeso y obesidad.

- $\quad$ El 30\% tenía un IMC con sobrepeso, el 18,7\% un IMC obeso y el 5,2\% un IMC mórbido. Un total del $54.5 \%$ de la población encuestada tiene sobrepeso, en comparación con el $65 \%$ de la población de los EE. UU.

- Del $71 \%$ de los encuestados que indicaron específicamente que su rol profesional incluía educación para la promoción de la salud, $22 \%$ son obesos (IMC> 30) y 32\% tienen sobrepeso (IMC 25-29.9).

- Aunque el 93\% de las enfermeras reconocen que el sobrepeso y la obesidad son diagnósticos que requieren intervención, el $76 \%$ no aborda el tema con pacientes con sobrepeso y obesos.

- Aproximadamente el $30 \%$ informó un título de asociado como el más alto grado académico obtenido, el $42 \%$ un título de bachillerato, el $25 \%$ un título de maestría y el $2 \%$ un doctorado.

- En términos del rol de la práctica actual, aproximadamente el $72 \%$ de los encuestados se describieron a sí mismos como una enfermera registrada, el $15 \%$ como una enfermera de práctica avanzada y el $5 \%$ como profesor 0 instructor de enfermería.

- $\quad$ El número medio de años en el papel actual fue de 9.9; La media de años en enfermería tenía 25 años. 


\section{TEMA}

Estrés, cambio de turno y comportamiento alimentario entre enfermeras en Arabia Saudita Central.

Ali M. Almajwal, MSc, PhD.

Arabia Saudita (2016)

\section{METODOLOGÍA EMPLEADA}

Estudio Transversal muestra: $\mathrm{N}=395$ enfermeras no sauditas

Instrumento:

*Cuestionario

Comportamiento de la Alimentación en Holanda (DEBQ)

*EI IMC se clasifica de acuerdo con el límite de la Organización Mundial de la Salud: 1) infraponderación (IMC <18.5), 2 ) Salud: 1 ) infraponderación (IMC $<18.5), 2)$
peso normal (IMC 18.5-24.9), 3 ) sobrepeso (IMC 25-29.9) y 4) obesos (IMC> 30).

*Statistical Package para el software de Ciencias Sociales versión 21.

\section{RESULTADOS CLAVES}

- Las enfermeras con estrés elevado tenían más probabilidades de presentarse con una alimentación restringida anormal (razón de posibilidades $[\mathrm{OR}]=1.52, \mathrm{p}=$ $0.004)$, emocional $(\mathrm{OR}=1.24 ; p=0.001)$ y externa $(\mathrm{OR}=1.21 ; p=0.001)$.

El trabajo de turno nocturno se asoció positivamente con la alimentación restringida $(O R=1.53 ; p=0.029)$ y la alimentación emocional $(O R=1.24 ; p=0.001)$, pero se asoció negativamente con la alimentación externa $(\mathrm{OR}=0.45 ; \mathrm{p}=0.001)$.

La mayoría de las enfermeras trabajaban en turno nocturno $(65,3 \%)$ más de 7 veces por mes $(40,3 \%)$

En esta muestra, casi el 50\% se clasificó como de IMC normal y el 50,6\% tenía sobrepeso $(37,7 \%)$ u obesidad $(12,9 \%)$

Se mostró además la asociación entre los estilos de alimentos, las variables socioeconómicas y de trabajo.

- El estrés y la educación se asociaron significativamente con todos los estilos de alimentación. Aquellos con alto estrés y con diplomas de pregrado eran más propensos a la alimentación restringida, la alimentación emocional y la alimentación externa que aquellos con bajo estrés y niveles educativos más altos.

- Aquellos que trabajaban en el turno de la noche tenían más probabilidades de experimentar una alimentación restringida, pero menos probabilidades de experimentar una alimentación externa.

- Además, el IMC y la frecuencia de los turnos nocturnos por mes se asociaron con una alimentación restringida.

- Las enfermeras con sobrepeso y las que trabajaban de 4 a 7 turnos de noche al mes estaban más inclinadas a comer de forma restringida en comparación con otros grupos.

- Los resultados indicaron que el estrés tenía una correlación significativa y positiva con todos los estilos de alimentación. Esto sugiere que las enfermeras con alto estrés tenían más problemas para comer.

- EI IMC fue significativo y se correlacionó positivamente con los estilos emocionales y estresantes.

- El análisis multivariado de la covarianza mostró una diferencia significativa entre los niveles de estrés y el trabajo por turnos para todos los estilos de alimentación.

- Las enfermeras que informaron de un alto estrés tuvieron un $52 \%$ más de probabilidades en la alimentación restringida anormal, un $24 \%$ más probabilidades en la alimentación emocional anormal, y un $21 \%$ más probabilidades en la alimentación externa en comparación con las enfermeras con bajo estrés. 
Conductas dietéticas deficientes entre enfermeras de hospitales en Seúl, Corea del Sur.

Kihye Han, $\mathrm{PhD}$

Smi Choi-Kwon, PhD Kyeong Sug Kim, PhD.

Corea del Sur (2015)
Estudio transversal Muestra: $\mathrm{N}=340$ enfermeras.

\section{Instrumento:}

* (KNHANES), que es una encuesta nacional que evalúa el estado de salud y nutrición de los coreanos desde 1998.

* Sobre la base de la recomendación para la clasificación del IMC en poblaciones asiáticas (Consulta de expertos de la OMS, 2004), se dividieron en cuatro grupos: bajo peso (IMC $<18.5 \mathrm{~kg} / \mathrm{m} 2$ ), peso normal (IMC 18.5-22.9 kg / m2), sobrepeso (IMC 23-24,9 kg / m2), y obesos (IMC $\geq 25 \mathrm{~kg} / \mathrm{m} 2$ ).

* IBM SPSS Statistics 21.0
- De las 340 enfermeras del estudio, el 74\% estaba involucrado en RSWN, el $10 \%$ en RSWON y el $16 \%$ en FS.

- En comparación con las enfermeras de RSWON y FS (es decir, sin noches), las enfermeras de RSWN tenían más probabilidades de ser más jóvenes, no estar casadas y vivir solas o con amigos, tener menos años de experiencia como enfermeras y trabajar en unidades de atención directa a pacientes (es decir, cuidados críticos o salas generales) (ps $<0.01$ ).

- La obesidad se diferenció significativamente según los horarios de trabajo ( $p=0.03$ ); Las RSWN tenían más peso que RSWON o FS.

- Las enfermeras con una mayor ingesta de alimentos de lo normal mientras estaban bajo estrés se encontraron con mayor frecuencia entre las personas con turnos rotativos ( $R S W N=74 \%$; $R S W O N=71 \%$ ) en comparación con las enfermeras de FS $(45 \%)(22=18.05, p=0.01)$.

- La cantidad de refrigerios bajo estrés varió según el horario de trabajo; El $72 \%$ de las enfermeras de RSWN y el $69 \%$ de las enfermeras de RSWON consumieron más de lo normal, mientras que el $45 \%$ de las enfermeras de FS lo hicieron $\left(\square^{2}=21.00, p<0.01\right)$. 


\section{TEMA}

Las asociaciones con estrés laboral, apoyo social y sobrepeso / obesidad entre enfermeras de hospitales.

Li Fang, Li-Ping Hsiao, Shu-Hui Fang \& Bao-Chen Chen

Taiwan (2018)

\section{METODOLOGÍA EMPLEADA}

Estudio transversal Muestra: $\mathrm{N}=237$

enfermeras.

\section{strumentos:}

La definición se utiliza para determinar la obesidad en la población asiática. Se describe a continuación: $24 \leqq$ IMC $<27$ como sobrepeso, IMC $\geq 27$ según la obesidad.

*Escala de Desequilibrio de EsfuerzoEsfuerzo (ERI) y la Escala de Apoyo Social.

*SPSS versión 15.0

\section{RESULTADOS CLAVES}

- La edad promedio de los participantes fue de 33.4 años (varió de 21 a 54 años) Treinta y una (13\%) enfermeras tenían sobrepeso u obesidad al momento de ser contratadas.

Bastantes participantes $(77,32 \%)$ experimentaron 1-2 días por semana de sueño de mala calidad, seguidos de 66 participantes (28\%) que tenían menos de 1 día por semana de sueño de mala calidad, 65 participantes (27\%) que tenían 34 días por semana, sueño de mala calidad, y 28 participantes (12\%) que tienen 5-7 días por semana sueño de mala calidad.

- Hubo $86(36.3 \%)$ encuestados que mostraron un exceso de compromiso con sus trabajos. Alrededor de un tercio de los participantes $(74,31 \%)$ mostraron "sobrepeso u obesidad".

- Se encontraron diferencias estadísticas significativas entre el sobrepeso y la obesidad de los participantes y su frecuencia de ejercicio, el tipo de tiempo de trabajo, la frecuencia de los turnos de noche y las horas de trabajo por semana.

- No hubo relaciones entre el sobrepeso / obesidad de los participantes y su edad, nivel educativo, sueño deficiente, estado civil, hábito de beber, hábito de fumar, año laboral, unidad de trabajo, puesto de trabajo y sobrepeso / obesidad a la edad de 20 años.

- El sobrepeso / obesidad de los participantes se asoció con el estrés laboral (P $<.01)$. Sin embargo, no hubo relaciones entre el estrés laboral de las enfermeras y su exceso de compromiso con su trabajo $(P=.772)$.

- El sobrepeso / obesidad de los encuestados se asoció altamente con el apoyo social $(\mathrm{t}=3.924, \mathrm{p}<.01)$. Hubo correlaciones significativas entre el sobrepeso / obesidad de los participantes y el grado de apoyo de sus familiares, amigos y colegas $(t=2.04, P=.04 ; t=2.74, P<.01 ; t=4.726, P<.01)$.

- El estudio mostró que la frecuencia del ejercicio regular, el tipo de trabajo, la frecuencia del turno nocturno, las horas de trabajo semanales $(44 \sim<48$ horas / $<44$ horas; $\geq 48$ horas / $<44$ horas), un alto estrés laboral ( $E$ / La proporción de $R>1$ ) y el apoyo social fueron predictores importantes del sobrepeso / obesidad de las enfermeras.

- Las enfermeras con ejercicio regular igual o inferior a 2 días ( $\leqq 2$ días) por semana fueron 8.21 veces $(\operatorname{Exp}(B)=0.122 ; \mathrm{P}=.010)$ más propensas a tener sobrepeso / obesidad que aquellas que con ejercicio regular igual o Más de 3 días ( $\geqq 3$ días) por semana. - Las enfermeras con estrés laboral alto fueron 5.76 veces (Exp (B) 5.764: $\mathrm{P}<0.01$ ) más propensas a tener sobrepeso / obesidad. 


\section{TEMA}

Association between shift work and obesity

among female nurses: Korean

Nurses' Survey

Min-Ju Kim, Kuk-Hui Son, HyunYoung Park, Dong-Ju Choi, Chang-

Hwan Yoon, Hea-Young Lee, Eun-Young Cho and Myeong-Chan Cho.

Corea (2013)

\section{METODOLOGÍA EMPLEADA}

Estudio transversal Muestra $\mathrm{N}=9.989$ de las cuales 5.287 trabajadores por turnos $\mathrm{y}$ 4.702 trabajadores sin turno.

\section{Instrumento:}

*EI IMC se calculó como el peso corporal

$(\mathrm{kg})$ dividido por Altura al cuadrado (m2).

Los umbrales de IMC fueron $\geq 23 \mathrm{~kg} / \mathrm{m} 2$ para el sobrepeso y $\geq 25 \mathrm{~kg} / \mathrm{m} 2$ para la obesidad.

* Los cuestionarios recopilaron información sobre características demográficas, comorbilidades, síntomas de enfermedades cardiovasculares, historia clínica, antecedentes familiares de enfermedades, salud reproductiva, estilo de vida, características psiquiátricas y ocupacionales, patrones dietéticos e ingesta de alimentos.

* SPSS Statistics 21.

\section{RESULTADOS CLAVES}

- Los trabajadores sin turno tenían un IMC medio más alto que los trabajadores con turno actual $(21.4 \pm 2.6 \mathrm{~kg} / \mathrm{m} 2 \mathrm{vs} .20 .5 \pm 2.5 \mathrm{~kg} / \mathrm{m} 2, \mathrm{P}<0.001)$.

- De todos los sujetos, la prevalencia de fumadores actuales fue relativamente baja $(1,2 \%)$ y casi la mitad de los participantes eran bebedores regulares.

- Los trabajadores de turno actuales tenían menos probabilidades de hacer ejercicio regularmente que los trabajadores sin turno (41.2\% vs. $47.3 \%)$.

- Los problemas de sueño durante 7 a 13 días o todos los días durante las últimas 2 semanas fueron más frecuentes entre los trabajadores por turnos (27.2\%). Hubo diferencias estadísticamente significativas en el estado de salud auto percibido de acuerdo con el trabajo actual $(P<0,001)$.

- Entre los trabajadores de turno actuales, los sujetos con la mayor duración del trabajo por turnos tenían más probabilidades de ser mayores $(P<0,001)$ y de tener un IMC más alto $(P<0,001)$. Esos sujetos también tenían más probabilidades de tener hipertensión, diabetes, hiperlipidemia o cáncer de mama $(P=0,003, P<0,001, P=0,003, P<0,001)$.

- La prevalencia global de sobrepeso u obesidad y obesidad fue de $18.6 \%$ y $7.4 \%$.

- Hubo correlaciones significativas y positivas entre la obesidad y la duración del trabajo por turnos.

- Los factores que se asociaron significativamente con la obesidad incluyeron la edad, el estado actual de fumar, el estado civil, la omisión del desayuno, el ejercicio regular y el estado de salud percibido por uno mismo 
Los factores ocupacionales asociados con la obesidad en el personal de enfermería son: escasa actividad física, horario laboral a tiempo completo, el trabajo en horario nocturno. Los trabajadores que tenían horario de trabajo diurno y a medio tiempo presentan mayor actividad física y menos IMC, por el contrario los que laboran a tiempo completo y en horarios nocturnos tienen menos posibilidad de realizar actividad física y presentan mayor IMC con preponderancia al sobrepeso el $31 \%$ de la muestra y $18 \%$ presentan obesidad (27). Otro factor asociado al sobrepeso y a la obesidad es la falta de sueño en trabajadores con horario nocturno, se socia la duración del sueño con el IMC a menor número de horas de sueño mayor es el IMC provocando aumento de peso y obesidad $(17,25)$.

La falta de tiempo para tomar descansos y consumir alimentos en horarios de trabajo de 12 horas, nocturnos o diurnos, están relacionados con altos IMC, el $59 \%$ de una población de enfermeras mayor de 47 años, en estas condiciones presentaron sobrepeso.

En dos grupos de trabajadores de enfermería, uno de turno diurno exclusivamente y el otro en turno rotativo se encontró que los puntajes para los contenidos de comidas y los patrones de alimentación temporales difirieron significativamente entre los grupos (p $<0.05$ ), lo que indica una dieta desequilibrada y un tiempo más irregular de comidas entre los trabajadores de turnos rotativos en comparación con los de horario diurno.

En una población de 13483 trabajadores la prevalencia de sobrepeso y obesidad fue mayor en el personal de enfermería que en otros profesionales de la salud; aunque más de la mitad de ese grupo tenían un IMC que indicaba sobrepeso (28).

En una población de 748 profesionales de la enfermería se encontró que la autoevaluación de la salud se encuentra determinada por la edad, Las personas entre 55 y 70 años tienen una salud autoevaluada negativa en comparación con las de 24 a 34 años quienes evalúan su salud de forma positiva.

En una población de 760 profesionales de la enfermería el $71 \%$ de los encuestados que indicaron específicamente que su rol profesional incluía educación para la promoción de la salud, $22 \%$ son obesos (IMC> 30) y $32 \%$ tienen sobrepeso (IMC 25-29.9). Aun cuando el $93 \%$ de las enfermeras reconocen que el sobrepeso y la obesidad son diagnósticos que requieren intervención, el 76\% no aborda el tema con pacientes con sobrepeso y obesos. Al tener sobrepeso se eximen de educar a los pacientes que se encuentran con sobrepeso u obesidad.

En una población de 340 enfermeras aquellas que presentaban un estrés elevado tenían más probabilidades de presentarse con una alimentación restringida anormal. El trabajo de turno nocturno se asoció positivamente con la alimentación restringida y la alimentación emocional. El $65,3 \%$ trabajan en horario nocturno y el 40,3\% más de 7 veces por mes. El 50,6 presenta sobrepeso $\mathrm{u}$ obesidad. Los resultados indicaron que el estrés tenía una correlación significativa y positiva con todos los estilos de alimentación. Lo que quiere decir que las enfermeras con alto estrés tenían más problemas para comer.

\section{Discusión}

Los altos índices de sobrepeso $y$ obesidad que presenta el personal de 
enfermería están relacionados con escasa actividad física, horario laboral a tiempo completo, el trabajo en horario nocturno.

El trabajo en el turno nocturno es una de las principales barreras para seguir una dieta saludable y practicar una actividad física adecuada. En este horario se presentan con mayor frecuencia patrones de consumo de comidas irregulares y falta de descansos en el trabajo, lo que conlleva a una inadecuada elección de los alimentos que se deben consumir.

Los turnos largos, con más de 8 horas y las pausas insuficientes no permiten una dieta saludable. Las demandas de trabajo durante un turno a veces hacen que sea difícil encontrar oportunidades para tomar un descanso.

En los hospitales la comida disponible no es balanceada. También existen carencias en la infraestructura porque no están adecuadas las instalaciones para apoyar la realización de una actividad física.

El personal de enfermería está consciente del papel de la nutrición en la salud pero las exigencias y condiciones de trabajo no les permiten hacer un cambio para mejorar su calidad de vida. Mencionan con frecuencia la falta de tiempo para preparar comidas saludables debido a las largas horas y al exceso de trabajo.

Los efectos de una inadecuada alimentación conllevan a alteraciones nutricionales como el sobrepeso y obesidad que es una afección grave que puede producir complicaciones; como; síndrome metabólico, presión arterial alta, ateroesclerosis, enfermedad del corazón, diabetes, niveles elevados de colesterol en sangre, distintos tipos de cáncer y hasta trastornos del sueño, asociándose al estrés laboral.

La duración corta de sueño, podría estar asociada con una reducción de la leptina y un aumento de la ingesta total de energía y grasa, lo que conlleva a alteraciones nutricionales y alimentarias en relación con los turnos nocturnos rotativos.

La demanda que se debe afrontar ante el cuidado directo del paciente y los turnos rotativos, conllevan a una sobrecarga de responsabilidad $\mathrm{y}$ deber para las enfermeras, reflejando angustia mental y alteraciones en el estado emocional, mismas que convergen en alteraciones de alimentación, sueño y descanso.

El personal de enfermería describe los beneficios percibidos por la participación en programas de incremento de la actividad física. Estos beneficios incluyen reducir el estrés y la fatiga en el trabajo, sentirse con energía antes del trabajo y relajado después del trabajo. Sin embargo, no siempre pudieron priorizar la participación en estos programas porque otros compromisos, como las actividades sociales y familiares se lo impidieron.

Trabajar largas horas y estar cansado fueron los principales factores que influyeron en el estrés laboral. Hay consenso en considerar que la falta de tiempo y tener compromisos familiares o sociales aumenta la responsabilidad, la carga laboral y la fatiga.

Los participantes relacionaron directamente el estrés laboral con los hábitos dietéticos. El estrés se relacionó con la alimentación emocional y la preponderancia al consumo de alimentos altos en grasa, sal y azúcar, como el chocolate, los dulces y la comida rápida. Aun cuando intentaron ser conscientes de su dieta, el estrés y la fatiga los conduce a la falta de autocontrol sobre la dieta.

El personal de enfermería que trabajan en el turno nocturno identificó con frecuencia el aumento de peso y el hecho de vivir con ENT, como la hipertensión, como 
sus principales preocupaciones de salud. La percepción es que el sobrepeso tiene un impacto negativo en el rendimiento laboral.

El dolor de espalda y la exposición a la tuberculosis (TB) y a otras enfermedades contagiosas son identificados como problemas de salud relacionados con la ocupación.

El género, la edad, la antigüedad en el trabajo, los turnos rotatorios, la cantidad de trabajo, el tipo de trabajo, el nivel de estrés, la calidad del sueño, la actividad física, el peso/la talla (IMC) son factores identificados que inciden en la salud nutricional del personal de enfermería.

El ambiente laboral a través de sus dependencias de RR.HH. y representaciones sindicales debe promover y apoyar las acciones que permitan conseguir la mejora de los hábitos de salud especialmente calidad nutricional, actividad física $\mathrm{y}$ manejo del estrés del personal de enfermería que se desempeña en hospitales.

\section{CONCLUSIONES}

$\mathrm{E}$ l método de revisión sistemática logró aproximar a la problemática, mediante el análisis de las principales investigaciones realizadas a nivel mundial, alcanzando identificar $\mathrm{y}$ establecer los factores que inciden en las afecciones nutricionales para el personal de enfermería de las áreas hospitalarias, que resultan perjudiciales al poderse desencadenar en enfermedades no transmisibles como la diabetes, hipertensión y la obesidad, enfermedades que a nivel mundial van en incremento, es por ello la importancia de promover estudios que contribuyan al cuidado del cuidador, al fomento de una vida laboral saludable y al derecho de trabajar bajo entornos que favorezcan la salud de todos.

\section{REFERENCIAS BIBLIOGRÁFICAS}

1. Organización Mundial de la Salud OMS y la Organización Panamericana de la Salud OPS. Estrategia de Promoción de la salud en los lugares de trabajo de América Latina y el Caribe: Anexo № 6 - Documento de Trabajo. Ginebra. (Suiza): Organización Mundial de la Salud; 2000.

2. Organización Mundial de la Salud (OMS). Recomendaciones mundiales sobre actividad física para la salud. Ginebra, Suiza: Organización Mundial de la Salud, 2010.

3. Organización Mundial de la Salud. Obesidad y sobrepeso. World Health Organization. 2018; Acceso: 19/10/2018. Disponible en: https://www.who.int/newsroom/fact-sheets/detail/obesity-andoverweight

4. Velazco J. Evaluación de la dieta en escolares de Granada. [Tesis doctoral en línea]. Universidad de Granada. 2008. Acceso: 22/10/2018. Disponible en: http://digibug.ugr.es/bitstream/handle/10 481/35891/17370036.pdf?sequence=1\&is Allowed=y

5. Pollack KM, Cheskin LJ. Obesity and workplace traumatic injury: does the science support the link? Inj Prev. 2007; 13(5):297-302.

6. Stansfeld SA, y Marmot MG. Stress and the Heart: Psychosocial Pathways to Coronary Heart Disease. London, England: BMJ Publishing Group; 2002.

7. Wallis DJ, y Hetherington MM. Emotions and eating. Selfreported and experimentally induced changes in food intake under stress. Appetite. 2009; 52(2):355-362.

8. Caruso CC, Bushnell T, Eggerth D, et al. Long working hours, safety, and health: toward a national research agenda. Am J Ind Med. 2006; 49(11):930-942. 
9. Faugier J, Lancaster J, Pickles D, et al. Barriers to healthy eating in the nursing profession: part 1. Nurs Stand. 2001; 15(36):33-36.

10. Faugier J, Lancaster J, Pickles $D$, et al. Barriers to healthy eating in the nursing profession: part 2. Nurs Stand. 2001; 15(37):33-35.

11. de Assis MA, Kupek E, Nahas MV, et al. Food intake and circadian rhythms in shift workers with a high workload. Appetite. 2003; 40(2):175-183.

12. Waterhouse J, Buckley P, Edwards B, y Reilly T. Measurement of, and some reasons for, differences in eating habits between night and day workers. Chronobiol Int. 2003; 20(6):1075-1092.

13. Burch JB, Tom J, Zhai Y, et al. Shiftwork impacts and adaptation among health care workers. Occup Med (Lond). 2009; 59(3):159-166.

14. Han K, Trinkoff AM., Storr C, GeigerBrown J. Job Stress and Work Schedules in Relation to Nurse Obesity. 2011; 41(11):488-495.

15. Straif K, Baan R, Grosse Y, Secretan B, El GF, Bouvard V, et al. Carcinogenicity of shift-work, painting, and fire-fighting. Lancet Oncol 2007; 8: 1065-1066. PMID: 19271347.

16. Wang XS, Armstrong ME, Cairns BJ, Key TJ, Travis RC. Shift work and chronic disease: the epidemiological evidence. Occup Med (Lond) 2011; 61: 78-89.

17. Zhao I, Bogossian F, Song S, Turner C. The association between shift work and unhealthy weight: a cross-sectional analysis from the Nurses and Midwives' e-cohort Study. J Occup Environ Med 2011; 53: 153158. PMID: 21270661.

18. Poulsen K, Cleal B, Clausen T, Andersen LL. Work, diabetes and obesity: a seven year follow-up study among Danish health care workers. PLoS One 2014; 9: e103425. Doi: 10.1371/journal.pone.0103425 PMID: 25068830.
19. Van DA, Boot C, Merkus S, Smid T, van der Beek A. The effects of shift work on body weight change- a systematic review of longitudinal studies. Scand J Work Environ Health. 2011; 37:263-275. Doi: 10. 5271/sjweh.3143 PMID: 21243319.

20. Kubo T, Oyama I, Nakamura T, Shirane $K$, Otsuka $H$, Kunimoto $M$, et al. Retrospective cohort study of the risk of obesity among shift workers: findings from the Industry-based Shift Workers' Health study, Japan. Occup Environ Med 2011; 68: 327-331. Doi: 10.1136/oem.2009.054445 PMID: 20884794.

21. Roos E, Lallukka $T$, Rahkonen $O$, Lahelma E, Laaksonen M. Working conditions and major weight gain-a prospective cohort study. Arch Environ Occup Health 2013; 68: 166-172. Doi: 10.1080/ 19338244.2012.686931 PMID: 23566324.

22. Geliebter A, Gluck ME, Tanowitz M, Aronoff NJ, Zammit GK. Work-shift period and weight change. Nutrition 2000; 16: $27-$ 29. PMID: 10674231.

23. Smith P, Fritschi L, Reid A, Mustard C. The relationship between shift work and body mass index among Canadian nurses. Appl Nurs Res 2013; 26: 24-31. Doi: 10.1016/j.apnr.2012.10.001 PMID: 23158849.

24. Manenschijn L, van Kruysbergen RG, de Jong FH, Koper JW, van Rossum EF. Shift work at young age is associated with elevated long-term cortisol levels and body mass index. J Clin Endocrinol Metab 2011; 96: 1862-1865

25. Beata Peplonska, Agnieszka Bukowska, Wojciech Sobala. Association of Rotating Night Shift Work with BMI and Abdominal Obesity among Nurses and Midwives. 2015; 10(7): 1-13.

26. Hutton B, Catalá-López F, y Moher, D. La extensión de la declaración PRISMA para revisiones sistemáticas que incorporan metaanálisis.: PRISMA-NMA. Med Clin 
(Barc). 2016. Acceso: 25/10/2018. among nurses in Scotland: A cross-sectional Disponible en: study using the Scottish Health Survey. http://dx.doi.org/10.1016/j.medcli.2016.0 2.025

27. Dal Lae China, Soohyun Namb, and SooJeong Leea. Occupational factors associated with obesity and leisure-time physical activity among nurses: Across sectional study Int J Nurs Stud. 2016 May; 57:60-69. Disponible en: doi:10.1016/j.ijnurstu.2016.01.009

International Journal of Nursing studies 53 October 2015. Acceso: 18/10/2018. Disponible en: DOI: 10.1016/j.ijnurstu.2015.10.015

Conflicto de interese: Los autores declaran que no existe conflicto de intereses para la publicación del presente artículo.

28. Kyle $R$, Neall $R$, $y$ Atherton, $L$.

Financiamiento: Autofinanciamiento Agradecimiento: No declaran Prevalence of Overweight and obesity 